April 2001

HIP-2001-09/TH

\title{
g-2 of the muon in SUSY Models with Gauge Multiplets in the Bulk of Extra-Dimensions
}

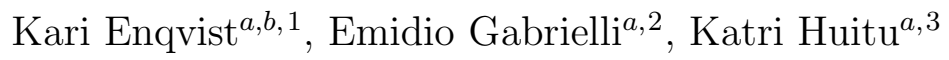 \\ ${ }^{a}$ Helsinki Institute of Physics, and \\ ${ }^{b}$ Physics Dept., University of Helsinki \\ $\mathrm{POB} 64$, \\ 00014 University of Helsinki, Finland
}

\begin{abstract}
We analyze the supersymmetric contributions to the anomalous magnetic moment of the muon $\left(a_{\mu}\right)$ in the framework of pure and gaugino-assisted anomaly mediation models, and gaugino mediation models. In the last two models the gauge multiplets propagate in the higher dimensional bulk, providing a natural mechanism for solving the problem of negative squared slepton masses present in the pure anomaly mediation models. In the light of the new BNL results for $a_{\mu}$, we found that the pure and gaugino-assisted anomaly mediation models are almost excluded by the BNL constraints at $2 \sigma$ level when combined with CLEO constraints on $b \rightarrow s \gamma$ at $90 \%$ of C.L. In contrast, the gaugino mediation models provide extensive regions in the SUSY parameter space where both of these constraints are satisfied.
\end{abstract}

\footnotetext{
${ }^{1}$ kari.enqvist@helsinki.fi

${ }^{2}$ emidio.gabrielli@helsinki.fi

${ }^{3}$ katri.huitu@helsinki.fi
} 
From the phenomenological point of view the breaking of supersymmetry (SUSY) is an essential issue in supersymmetric model building, since it will determine the soft mass parameters, and therefore the mass pattern of the model. After the detection of a few supersymmetric partners of ordinary particles much can be deduced of the breaking mechanism. Before direct detection, one can get indirect information from the low energy signals. In this respect, the measurement of the anomalous magnetic moment of the muon $\left(a_{\mu}\right)$ is a golden test of the SUSY contributions beyond the Standard Model (SM).

Recently, the new measurement of $a_{\mu}$ at Brookhaven E821 experiment [1], has reported a $2.6 \sigma$ deviation from the $\mathrm{SM}$ prediction [2],

$$
a_{\mu}(\mathrm{E} 821)-a_{\mu}(S M)=(43 \pm 16) \times 10^{-10},
$$

although this deviation has been criticized [3], mainly due to the large theoretical uncertainties affecting the hadronic contributions to the vacuum polarization' However, the possibility that new physics is responsible for such a deviation, has motivated an intense theoretical activity especially in the framework of supersymmetric theories [6]. Indeed, if this deviation has a supersymmetric origin, the lightest SUSY particles should be in the expected discovery range of the Large Hadronic pp Collider (LHC) at CERN and even of the Fermilab $2 \mathrm{TeV} p \bar{p}$ collider.

The aim of the present paper is to analyze, in the light of the BNL deviation, the predictions for $a_{\mu}$ in a special class of supersymmetric models, where the SUSY breaking is realized with gauge multiplets propagating in the bulk of extra dimensions. In recent years several interesting aspects of supersymmetry breaking have emerged. Especially the branes, which are typical in models with extra dimensions, have been found to fit naturally with the idea of breaking supersymmetry in a hidden sector.

In this framework, the anomaly mediated breaking (AMSB) [7] is particularly attractive, since this contribution to the breaking will always be present. Unfortunately this mechanism has a well known flaw: squares of the slepton masses become negative. Because of the other virtues of the model, several methods have been proposed in order to remove this problem [8]. Assuming gauge multiplets in the higher dimensional bulk, it was found in [9] that at one loop the squared slepton masses obtain contributions, which would be of the correct size for solving the slepton mass problem.

Another breaking mechanism connected with the extra dimensions is the so-called gaugino mediation ( $\tilde{g} \mathrm{MSB})$ [10], in which the gauge superfields propagate in the bulk

\footnotetext{
${ }^{4}$ In reference [1] it has been stressed that, although some previous SM calculations agree with the experimental result for $a_{\mu}$ [5], they are not on a equal footing or up-to-date in comparison with the analysis of Davier and Höcker in [2].
} 
in addition to gravity, and couple at tree-level to a singlet at SUSY breaking brane. This mechanism does not have any problems with experiments, but it has some arbitrariness in the scale of the compactification radius.

We will work in the framework proposed by Randall and Sundrum in [7], where two branes are located in one extra dimension. One of the branes contains the hidden sector, while the other brane contains the ordinary matter. Gravity is in the bulk. Here we will assume that also the gauge supermultiplets reside in the bulk, following [9, 10.

If there is no coupling of the gauge fields to the fields in the hidden sector, the model is the one presented in [9], in which the squared scalar masses, in addition to the usual contributions from superconformal anomaly, receive contributions at one loop due to the corrections to the wave function renormalization of gauginos.

In pure anomaly mediation the soft supersymmetry breaking parameters are obtained as [7]

$$
\begin{aligned}
M_{a} & =\frac{\beta_{g_{a}}}{g_{a}} m_{3 / 2} \\
\tilde{m}_{i}^{2} & =-\frac{1}{4}\left(\frac{\partial \gamma_{i}}{\partial g} \beta_{g}+\frac{\partial \gamma_{i}}{\partial Y} \beta_{Y}\right) m_{3 / 2}^{2} \\
A_{Y} & =-\frac{\beta_{Y}}{Y} m_{3 / 2}
\end{aligned}
$$

where $\beta_{i}$ and $\gamma_{i}$ are the beta functions and anomalous couplings, respectively. The explicit expressions can be found in [11]. It is obvious that the slepton mass squared containing positive $S U(2) \beta$-functions are negative. The extra contribution $\Delta \tilde{m}_{i}^{2}$ to $\tilde{m}_{i}^{2}$ in (2), received in the framework of [9], is given by

$$
\Delta \tilde{m}_{i}^{2}=2 \zeta(3) \Gamma(4) \sum_{a} C_{a}(i) \frac{g^{2}}{16 \pi^{2}} \frac{1}{\left(M_{*} L\right)^{2}} m_{3 / 2}^{2} \equiv \eta \sum_{a} C_{a}(i) \frac{g^{2}}{16 \pi^{2}} m_{3 / 2}^{2},
$$

where $\sum_{c} C_{a}(i)$ is the weighted sum over the quadratic Casimir for the $i$ matter scalar representations, tabulated in [9], and $g$ is the gauge coupling at unification scale. The Casimir factors will give to the scalar masses a nonuniversal contribution, which is the major difference between the gaugino-assisted and minimal anomaly mediation models. In the latter, $\Delta \tilde{m}_{i}^{2}$ is assumed universal. From the requirement of small flavour violating operators [9], the volume factor $M_{*} L \gtrsim 16$. Thus the numerical value of $\left(M_{*} L\right)^{-2} \sim g^{2} /\left(16 \pi^{2}\right)$. On the other hand the scalar masses are two-loop suppressed in pure anomaly mediation, in contrast to the one-loop suppression in Eq. (3). Accidentally, therefore, the extra contribution to the scalar masses is of the correct size to solve the slepton mass problem in AMSB. $\eta$ will be used as the parameter in our numerical calculations. 
If there is a direct coupling of gauge fields to the SUSY breaking brane, with a singlet which receives a VEV, the gauginos get a SUSY breaking mass [10]. Minimally the gaugino mediation model has three parameters, namely the Higgs mixing parameter $\mu$, the common gaugino mass $M_{1 / 2}$ and the compactification scale $M_{c}$. Following Schmaltz and Skiba in [10], we assume that at the GUT scale the soft breaking $A$ parameters, as well as the soft scalar masses, vanish and the compactification scale is in the range $M_{G U T} \lesssim M_{c} \lesssim M_{\text {Planck }} / 10$. However, since we are interested in analyzing a more general scenario than in [10], we will take $\tan \beta$ as a free parameter. This is effected by relaxing the condition of vanishing soft $B$ parameter at GUT scale assumed in [10]. The main reason for our choice is that, as shown in the following, the SUSY contribution to $a_{\mu}$ is very sensitive to $\tan \beta$.

In gaugino mediation models the gaugino mass is proportional to the $F$ component of a chiral superfield on the SUSY breaking brane. To be more specific, with a singlet $S$, the gaugino masses are generated by

$$
\int d^{2} \theta \frac{S}{M_{*}^{2}} W^{\alpha} W_{\alpha} \delta(y-L)
$$

where $y$ is the extra dimensional coordinate, $L$ is the place of the SUSY breaking brane, and the resulting gaugino mass is $M_{1 / 2} \propto F_{S} /\left(M_{*}^{2} L\right)$. If the $\mathrm{VEV} F_{S}$ does not exist, the gaugino masses could be generated by some higher dimensional operators, containing charged fields $\Sigma$ in extra dimensions,

$$
\int d^{4} \theta \frac{\Sigma^{\dagger} \Sigma}{M_{*}^{2}} W^{\alpha} W_{\alpha} \delta(y-L)
$$

giving a contribution of order $F_{\Sigma}^{2} /\left(M_{*}^{4} L\right)$. Assuming that $F_{S}$ and $F_{\Sigma}$ are of the same order, the anomaly induced contribution is negligible. However, if the magnitudes of $F_{S}$ and $F_{\Sigma}$ differ considerably, or one of them does not exist, it is of interest to study the situation in which one of these is dominant.

Now we analyze the SUSY contributions to $a_{\mu}$ in the framework of the anomaly and gaugino mediation models. The minimal supersymmetric standard model (MSSM) contributes to $a_{\mu}$ mainly via magnetic-dipole penguin diagrams, with an exchange of a chargino or a neutralino in the loop. As is well known, the $a_{\mu}$ is enhanced by $\tan \beta$, which is defined as $\tan \beta=\frac{\left\langle H_{U}\right\rangle}{\left\langle H_{D}\right\rangle}$. In the large $\tan \beta$ limit, the $a_{\mu}^{\mathrm{SUSY}}$ is dominated by the chargino diagram which is given by 18

$$
a_{\mu}^{\mathrm{SUSY}} \approx \frac{3 \alpha_{2}}{4 \pi} \tan \beta \frac{m_{\mu}^{2} \mu M_{2}}{m_{\tilde{\nu}}^{4}} F\left(x_{M_{2}}, x_{\mu}\right),
$$

where $M_{2}$ is the weak gaugino mass, $m_{\tilde{\nu}}$ is the sneutrino mass and $F$ is a loop function defined as

$$
F(x, y)=\frac{f(x)-f(y)}{x-y}, \quad f(x)=\frac{3-4 x+x^{2}+2 \log (x)}{3(1-x)^{3}}
$$


where $x_{M_{2}}=M_{2}^{2} / m_{\tilde{\nu}}^{2}, \quad x_{\mu}=\mu^{2} / m_{\tilde{\nu}}^{2}$.

From Eq.(6) we see that the free parameters entering in the $a_{\mu}^{\mathrm{SUSY}}$ formula at large $\tan \beta$ are the $\mu$ parameter, the sneutrino mass $m_{\tilde{\nu}}$, the weak gaugino mass $M_{2}$, and $\operatorname{sign}\left(\mu M_{2}\right)$. In addition, the sub-dominant neutralino diagram contribution depends also on the smuon and neutralino masses. However, in our analysis we have used the exact one-loop expression for $a_{\mu}^{\mathrm{SUSY}}$ which could be found, for instance, in Ref. [12].

The ingredients used in our analysis can be summarized as follows. The SUSY masses and parameters at electroweak scale are obtained from their boundary conditions at GUT scale, by solving the one-loop renormalization group equations of MSSM [13]. Moreover, we compute the $\mu$ parameter by requiring the condition of the correct electroweak symmetry breaking. In the computation of gaugino masses at low energy, we have included the next-to-leading order corrections in $\alpha_{s}$ and the top Yukawa coupling [11]. Indeed these corrections may become relevant for the anomalymediation scenarios, where the triplet of Winos $\left(\tilde{W}^{ \pm}, \tilde{W}^{0}\right)$ is nearly degenerate. Also in the calculation of the lightest Higgs mass $m_{h}$ we have included the leading one-loop radiative corrections [14.

In order to avoid tachyons and vacuum instabilities, we require that all the scalar masses at the GUT scale are positive. Besides, the present lower bounds on the SUSY particle masses from accelerator experiments have been imposed. In particular for the lightest chargino and Higgs masses, we use $m_{\chi^{ \pm}}>83 \mathrm{GeV}$ and $m_{h}>114 \mathrm{GeV}$, respectively. Note that our lower bound on chargino mass is suitable for the class of models considered here, see Ref. [15] for further details.

In addition, we have imposed the CLEO bounds on the decay $B \rightarrow X_{s} \gamma$ at $90 \%$ C.L. 16]

$$
2.0 \times 10^{-4}<\operatorname{BR}\left(B \rightarrow X_{s} \gamma\right)<4.5 \times 10^{-4}
$$

In MSSM, the SUSY contribution to $b \rightarrow s \gamma$ comes from the flavour-changing magneticdipole penguin diagrams with exchanges of charged Higgs, chargino, neutralino, and gluino [17. When the scalar soft-breaking terms at GUT scale are universal in flavour, such as for instance in the models considered here, the main contributions come from the diagrams with an exchange of charged Higgs and charginos. In our analysis we used the parametrization of [18] for the SUSY contribution to the $B \rightarrow X_{s} \gamma$ decay, which includes the next-to-leading order QCD corrections for the SM contribution, and the leading-order SUSY contributions to the relevant Wilson coefficients. []

\footnotetext{
${ }^{5}$ We have used in our analysis the central value for the SM branching ratio $B R\left(B \rightarrow X_{s} \gamma\right)=$ $3.28 \times 10^{-4}$. Note that in Ref. 18 the corresponding one was a bit higher, due to some (percentlevel) non-pertubative corrections included in that evaluation.
} 

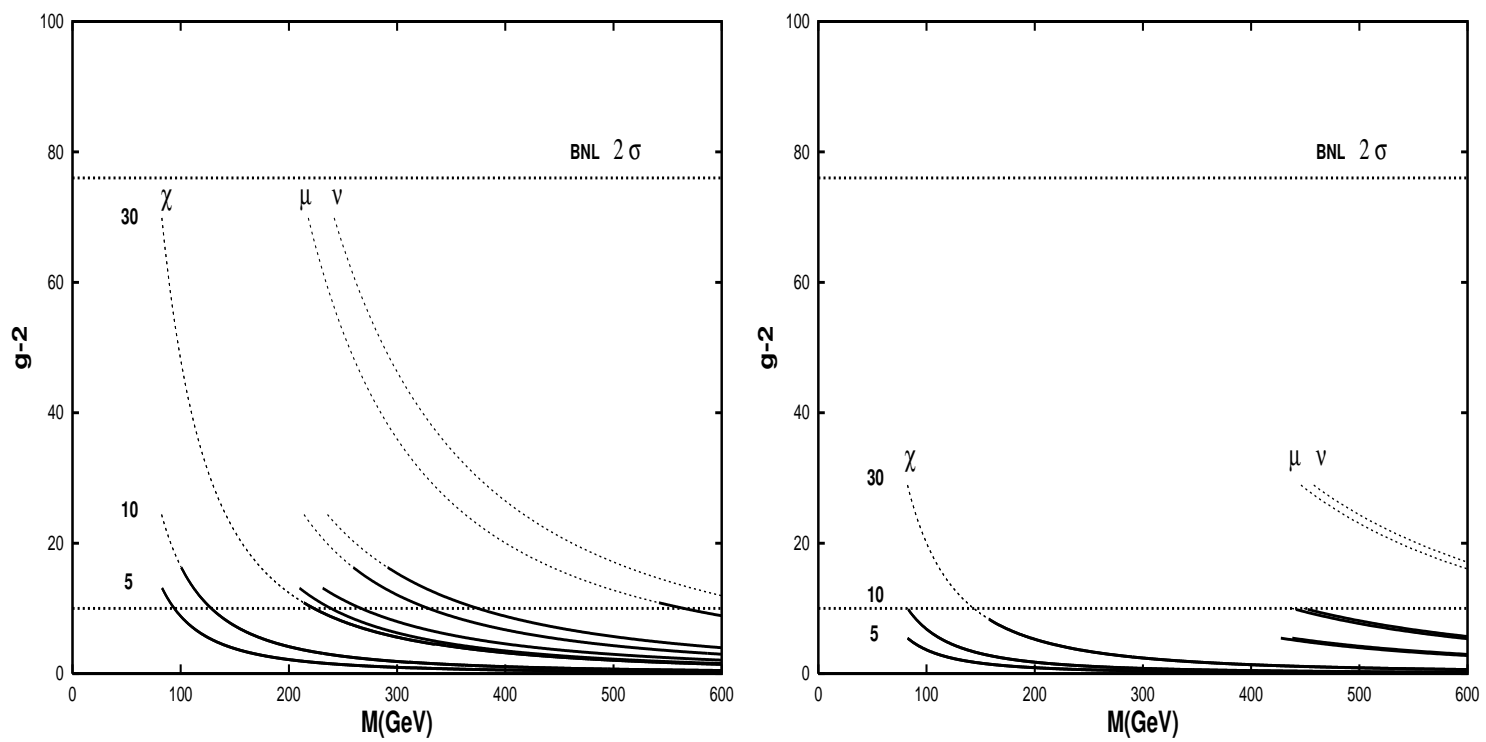

Figure 1: In the minimal anomaly mediation model, $a_{\mu}^{\text {SUSY }}$ (in units of $10^{-10}$ ) versus the lightest chargino $(\chi)$, smuon $(\mu)$, and sneutrino $(\nu)$ masses, for three values of $\tan \beta=$ $5,10,30$. The left and right plots correspond to $\eta=0.03$ and $\eta=0.1$ respectively, where $\eta$ is defined as in Eq.(3) with $\sum_{a} C_{a}(i)=1$. The area inside the two horizontal lines stands for the BNL deviation at $2 \sigma$ level. The dashed lines represent regions excluded by the $b \rightarrow s \gamma$ constraints.

As known, the $b \rightarrow s \gamma$ constraint plays a crucial role in the $a_{\mu}^{\mathrm{SUSY}}$ analysis, in particular at large $\tan \beta$. The reason is that in MSSM the chargino contribution to the $b \rightarrow s \gamma$ amplitude is enhanced by $\tan \beta$, and at large $\tan \beta$ it might become comparable in magnitude with the charged Higgs one, when chargino masses are quite light [17]. Moreover the charged Higgs contribution has always a positive interference with the SM amplitude, while the sign of the chargino contribution crucially depends on $\operatorname{sign}(\mu)$ (when the sign of $M_{2}$ is fixed). T Therefore $\operatorname{sign}(\mu)$ plays a decisive role in selecting the regions where the chargino and charged Higgs have constructive or distructive interferences, disfavoured or favoured by the CLEO constraints, respectively.

An important consequence of the BNL constraints on MSSM is that the value of $\operatorname{sign}(\mu)$ for which $a_{\mu}^{\text {SUSY }}$ becomes negative is not allowed. When the BNL constraints on $a_{\mu}$ are combined with the CLEO ones on $b \rightarrow s \gamma$, then there is no more freedom in choosing $\operatorname{sign}(\mu)$ in order to favour these last ones. This effect might strongly reduce the allowed SUSY parameter space. As shown in the following this will be the case for the anomaly mediation models.

\footnotetext{
${ }^{6}$ In some models the sign of $M_{2}$ is also a free parameter and therefore the physical sign in this case is given by $\operatorname{sign}\left(\mu M_{2}\right)$.
} 

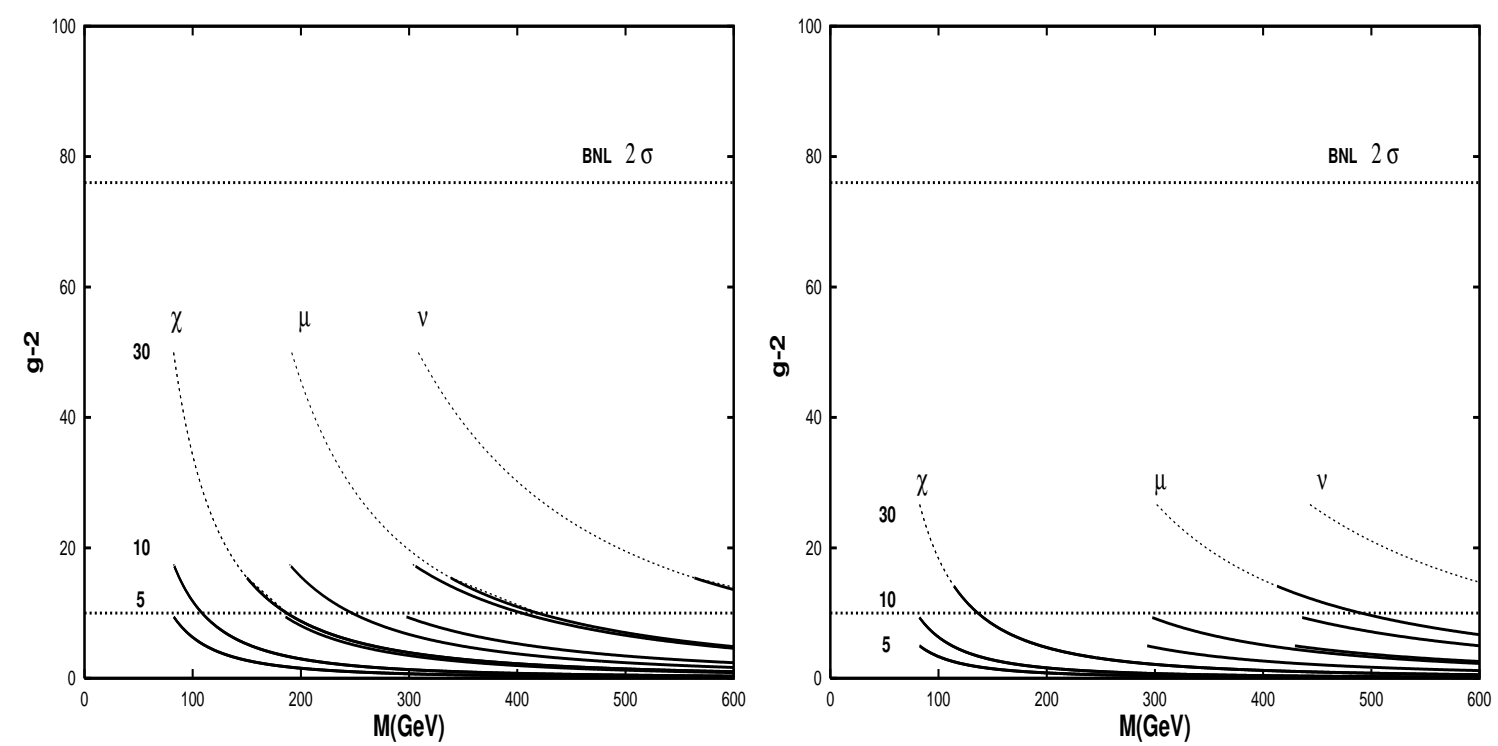

Figure 2: As in Fig. (1), but for the gaugino-assisted anomaly mediation model, where $\eta$ is defined in Eq.(3). The left and right plots correspond to $\eta=0.05$ and $\eta=0.1$ respectively.
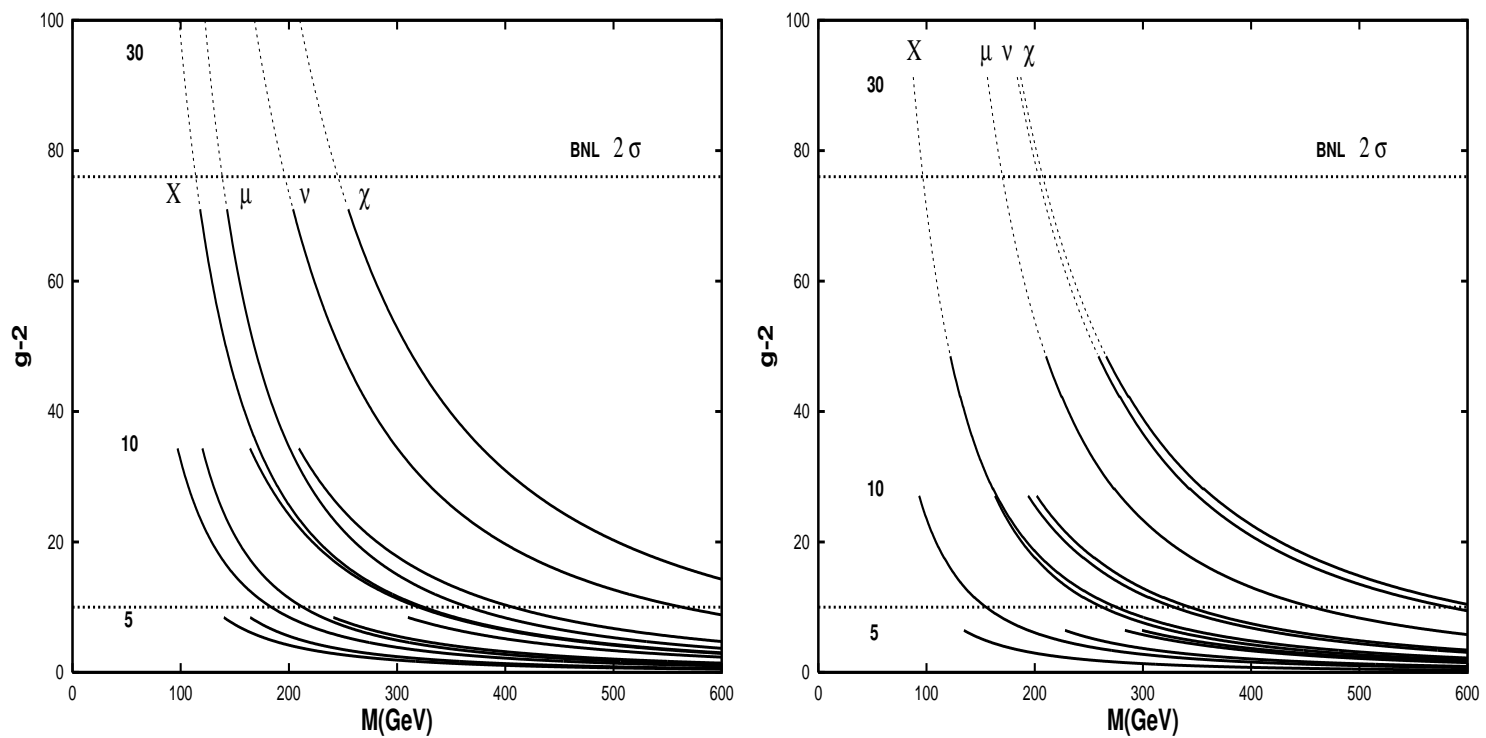

Figure 3: As in Fig. (11), but for the minimal gaugino mediation model with $S U(5)$ unified group. The label on the curves stand for the lightest neutralino $(X)$, chargino $(\chi)$, smuon $(\mu)$, and sneutrino $(\nu)$. The left and right plots correspond to $t_{c}=1$ and $t_{c}=4$ respectively, where $t_{c}=\log \left(M_{c} / M_{G U T}\right)$. 

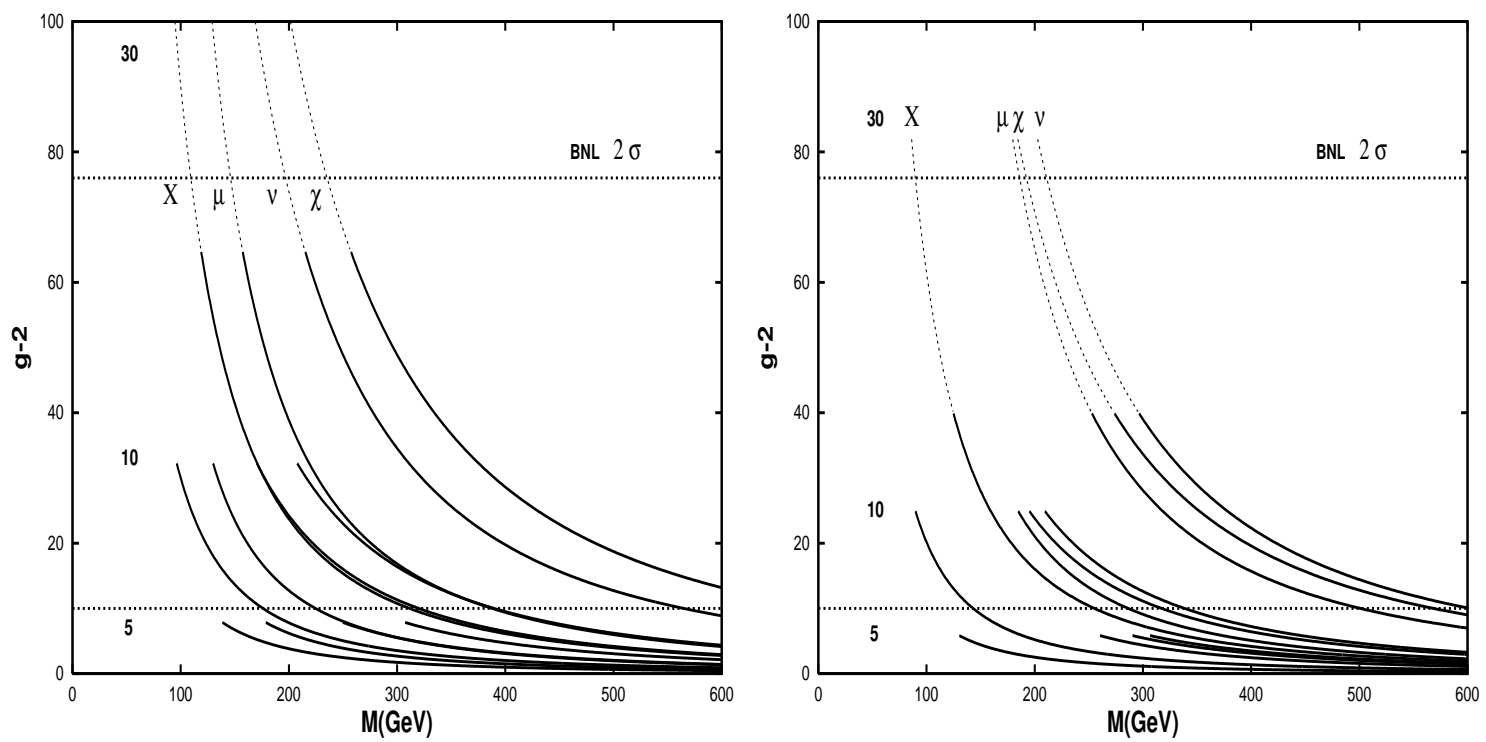

Figure 4: As in Fig. (3), but with $S O(10)$ unified group. The left and right plots correspond to $t_{c}=1$ and $t_{c}=4$ respectively.

Our numerical results are shown in Figs. (112), for the anomaly mediation and gaugino assisted anomaly-mediation respectively, and in Figs. (3- (3) for the minimal gaugino mediation models. In all these figures the region inside the two horizontal lines indicate the BNL deviation in Eq.(11) at $2 \sigma$ level. The $a_{\mu}^{\text {SUSY }}$ has been plotted versus the relevant low energy spectrum, such as the lightest chargino, neutralino, smuon, and sneutrino masses. We show the curves only for three representative values of $\tan \beta=5,10,30$, where the dashed lines always indicate the regions excluded by the $b \rightarrow s \gamma$ constraints.

Let us first discuss the results in the anomaly mediation scenarios. In Figs. (표), the curves corresponding to the lightest neutralino or chargino roughly coincide since in these models the lightest chargino and neutralino masses are almost degenerate, so we show only the chargino ones. In the minimal anomaly mediation scenario we parametrize $\tilde{m}^{2}$ as appearing in Eq.(3), but with $\sum_{a} C_{a}(i)=1$.

The left and right plots in Fig. (1) correspond respectively to $\eta=0.03$ and $\eta=0.1$. In particular, the value of $\eta=0.03$ corresponds to the allowed minimum for $\tilde{m}$ (at fixed $m_{3 / 2}$ ) for which no tachyons in the slepton sector will appear. The larger the $\eta$ is, the larger the SUSY scalars become at fixed chargino or neutralino masses. From the results of Fig. (11) one can see that already at $\tan \beta=30$ the combined effect of BNL and CLEO bounds exclude the minimal anomaly mediation scenario, while at intermediate $\tan \beta$ regions, such as $\tan \beta=10$, this model is barely allowed. We note that the lower bounds on the stau and lightest Higgs mass prevent the chargino mass going below $200 \mathrm{GeV}$ for $\tan \beta=30$. 
Clearly, when the scalar masses are increasead, $\eta=0.1$, the regions at moderate $\tan \beta$ are also excluded due to the SUSY decoupling, as can be seen comparing the left and right plots in Fig. (位). Then the main conclusion from these results is that the anomaly mediation models seem to be disfavoured in explaining the $2 \sigma$ BNL deviation, while preserving the CLEO bounds in Eq. (8). These results are also in agreement with the corresponding ones in [19] and in the second reference of [6].

In Fig. (2) we show the corresponding results in the gaugino-assisted anomaly mediation model. This model differs from the minimal anomaly-mediation one only by the fact that here the extra contributions to the scalar masses in Eq. (3) are not universal for all the scalars. As in Fig. (1),$\eta=0.05$ correspond here to the minimal allowed value for $\tilde{m}$, (at fixed $m_{3 / 2}$ ). Note that the minimum value for $\eta$ differs from the previous scenario due to the presence of the Casimir factors in Eq.(33). Also in this scenario we get similar results with respect to the minimal anomaly mediation one. From the results in Fig. (22) we see that the non-universality in the scalar sector does not help very much in getting allowed extensive regions where the CLEO and BNL constraints are satisfied. However, we see that, with respect to the minimal anomaly mediation scenario, also in this case the intermediate $\tan \beta$ regions, such as $\tan \beta=10$, are barely favoured in satisfying these constraints.

In Fig. (3) we show the results for the minimal gaugino mediation model with $\mathrm{SU}(5)$ unified group. In this scenario the free parameters are the common gaugino mass $M$, the parameter $t_{c}=\log \left(M_{c} / M_{G U T}\right)$, where $M_{c}$ is the compactification scale, $\tan \beta$, and $\operatorname{sign}(\mu)$. Since the scalar masses $\tilde{m}$ and trilinear couplings $A$ are radiatively generated through the renormalization group running from the compactification scale down to the GUT scale, they will be proportional respectively to $\tilde{m}^{2} \propto M^{2} \alpha t_{c}$, and $A \propto M \alpha t_{c}$ times averaged Casimir factors. For the exact expressions we have used the results of the last reference in [10]. In Fig. [3] we have shown two representative examples with $t_{c}=1$ and $t_{c}=4$ for the left and right plot respectively, and for $\tan \beta=5,10,30$. For dashed and continuous curves we use the same convention adopted in Figs. (15-2). From Fig. (3) we see that this class of models are favoured in obtaining extensive regions in the parameter space where $a_{\mu}^{\mathrm{SUSY}}$ is within the $2 \sigma \mathrm{BNL}$ deviation, while respecting the CLEO constraints, even at intermediate $\tan \beta$ regions $(\tan \beta>10)$. Moreover the regions with $\tan \beta<5$ seem to be excluded by the lower bounds of the $2 \sigma$ BNL constraints. Note that, with respect to the anomaly mediation models, the hierarchy of the SUSY particles masses plotted in Fig. (3) is changed. Now the neutralino is the lightest one, followed in order by the smuon, sneutrino, and chargino ones. Increasing the value of compactification scale, the lightest chargino and sneutrino masses become more degenerate, as shown in the right plot of Fig. (3). 
In Fig. (4), the results for the minimal gaugino mediation model with the $\mathrm{SO}(10)$ unified group are shown. The difference with the corrseponding results in Fig. (3) lies in the different boundary conditions at GUT scale [10]. As in Fig. (团) we see that also for this model there are extensive regions in the parameter space where both BNL and CLEO constraints are satisfied. Besides, as in the case of SU(5), the regions with $\tan \beta<5$ are excluded by the lower bound of the $2 \sigma$ BNL constraints. Note that when $t_{c}=4$ the sneutrino becomes heavier than the lightest chargino one, for the region of masses and $\tan \beta$ considered in Fig. (四).

Thus the remarkable aspect of the BNL deviation is that at $2 \sigma$ level one can obtain upper limits on the lightest chargino $\left(m_{\chi^{ \pm}}\right)$, neutralino $\left(m_{\chi^{0}}\right)$, smuon $\left(\tilde{m}_{\mu}\right)$, and sneutrino $\left(\tilde{m}_{\nu_{\mu}}\right)$ masses. The correspondig (conservative) upper limits in the minimal gaugino mediation can be summarized as follows. In the case of SU(5) we obtain, for $t_{c}=1$ (4) and $\tan \beta \leq 30$, the following results $m_{\chi^{0}} \leq 320$ (280) $\mathrm{GeV}, m_{\chi^{ \pm}} \leq 720$ (610) GeV, $\tilde{m}_{\mu} \leq 370$ (460) GeV, and $\tilde{m}_{\nu_{\mu}} \leq 560$ (580) GeV, while the corresponding ones for $\mathrm{SO}(10)$ are given by $m_{\chi^{0}} \leq 310(250) \mathrm{GeV}, m_{\chi^{ \pm}} \leq$ 690 (570) GeV, $\quad \tilde{m}_{\mu} \leq 390$ (500) GeV, and $\tilde{m}_{\nu_{\mu}} \leq 560$ (600) GeV. As we can see, these upper limits are not very sensitive to the different structure of $\mathrm{SU}(5)$ and $\mathrm{SO}(10)$ unified gauge group.

In this framework we have also analyzed the implications of the gaugino mediation scenarios for current dark matter detectors, DAMA [20] and CDMS [21]. Indeed it is known that the lightest neutralino is the natural candidate for dark matter in SUSY models with conserved $\mathrm{R}$ parity. These detectors are sensitive to the neutralinonucleon cross section $\left(\sigma_{\chi}\right)$ in the range of $10^{-6}-10^{-5} \mathrm{pb}$. Recently in the framework of MSSM with universal boundary conditions it was pointed out that the large $\tan \beta$ regime allows $\sigma_{\chi}$ to reach the above range [22]. In addition, with non-universal soft scalar masses it is possible to obtain large cross sections even for moderate $\tan \beta$ regions [23]. We checked that in the minimal gaugino mediation scenario, where non-universal soft masses are naturally generated at GUT scale [10], the neutralinonucleon cross section is unfortunately not enhanced. In particular we found that in both $S U(5)$ and $S O(10)$ models and in the parameter region where $a_{\mu}^{\mathrm{SUSY}}$ is positive, $\sigma_{\chi}$ is always below $10^{-7} \mathrm{pb}$ for $\tan \beta \leq 30$ and $1<t_{c}<4$.

In conclusion, assuming that the complete effect of the BNL deviation is due to supersymmetry, we find that the minimal and gaugino-assisted anomaly mediation models, seem to be both disfavoured by the BNL constraints at $2 \sigma$ level when combined with the CLEO constraints on $b \rightarrow s \gamma$ at $90 \%$ of C.L. On the contrary, in the minimal gaugino mediation models we found extensive regions in the SUSY parameter space, where these constraints are satisfied. Thus for these models, we have obtained 
conservative upper bounds on the relevant SUSY spectrum. These are of the order of $300 \mathrm{GeV}$ and $700 \mathrm{GeV}$ for the lightest neutralino and chargino respectively, and of the order of $500 \mathrm{GeV}$ and $600 \mathrm{GeV}$ for the lightest smuon and sneutrino respectively, when $\tan \beta \leq 30$ and the compactification scale is in the range $1 \leq t_{c} \leq 4$.

\section{Acknowledgements}

This work was partially supported by the Academy of Finland (project nos. 35224, 48787, and 163394).

\section{References}

[1] H.N. Brown et al. (Muon ( $g-2)$ Collaboration), Phys.Rev.Lett. 86, 2227 (2001).

[2] A. Czarnecki and W.J. Marciano, hep-ph/0102122; M. Davier and A. Höcker, Phys. Lett. B435, 427 (1998); M. Davier, hep-ex/9912044.

[3] F.J. Ynduráin, hep-ph/0102312.

[4] W.J. Marciano and B. Lee Roberts, hep-ph/0105056.

[5] A. Casas, C. López, and F.J. Ynduráin, Phys. Rev. 32, 736 (1985); K. Adel and F. J. Ynduráin, Rev. Acad. Ciencias (Esp.), 92 (1998); S. Eidelmann and F. Jegerlehner, Z. Phys. C67, 585 (1995).

[6] E.A. Baltz and P. Gondolo, hep-ph/0102147; J. Feng and K.T. Matchev, Phys. Rev. Lett. 86, 3480 (2001); L. Everett, G.L. Kane, S. Rigolin, and L.T. Wang, Phys. Rev. Lett. 86, 3484 (2001); T. Ibrahim, U. Chattopadhyay, and P. Nath, hep-ph/0102324; R. Arnowitt, B. Dutta, B. Hu and Y. Santoso, Phys. Lett. B505, 177 (2001); K. Choi, K. Hwang, S.K. Kang, K.Y. Lee, and W.Y. Song, hep-ph/0103048; A. Dedes and H. E. Haber, JHEP 0105, 006 (2001); S. Martin and J.D. Wells, hep-ph/0103067; S. Komine, T. Moroi, and M. Yamaguchi, Phys. Lett. B507, 224 (2001); H. Baer, C. Balázs, J. Ferrandis, and X. Tata, hep-ph/0103280; S. Baek, P. Ko, and H.S. Lee, hep-ph/0103218. 
[7] L. Randall, R. Sundrum, B557, 79 (1999); G.F. Giudice, M.A. Luty, H. Murayama, R. Rattazzi, JHEP 9812, 027 (1998) ; J.A. Bagger, T. Moroi, E. Poppitz, JHEP 0004, 009 (2000).

[8] A. Pomarol, R. Rattazzi, JHEP 9905, 013 (1999); R. Rattazzi, A. Strumia, J.D. Wells, B576, 3 (2000); Z. Chacko, M. Luty, E. Pontón, Y. Shadmi, Y. Shirman, hep-ph/0006047; E. Katz, Y. Shadmi, Y. Shirman, JHEP 9908, 015 (1999); Z. Chacko, M.A. Luty, I. Maksymsk, E. Pontón, JHEP 0004, 001 (2000); I. Jack, D.R.T. Jones, B482, 167 (2000); M. Carena, K. Huitu, T. Kobayashi, B592, 164 (2001).

[9] D.E. Kaplan, G.D. Kribs, JHEP 0009, 048 (2000).

[10] D.E. Kaplan, G.D. Kribs, M. Schmaltz, Phys. Rev. D62, 035010 (2000); Z. Chacko, M.A. Luty, A.E. Nelson, E. Ponton, JHEP 0001, 003 (2000); M. Schmaltz, W. Skiba, Phys.Rev. D62, 095005 (2000).

[11] T. Gherghetta, G.F. Giudice, J.D. Wells, Nucl. Phys. B559, 27 (1999).

[12] U. Chattopadhyay and P. Nath, Phys. Rev D53, 1648 (1996); T. Moroi, Phys. Rev D53, 6565 (1996); 56, 4424(E) (1997); M. Carena, G.F. Giudice, and C.E.M. Wagner, Phys. Lett B390, 234 (1997); U. Chattopadhyay, D.K. Ghosh, and S. Roy, Phys. Rev. D62, 115001 (2000); K.T. Mahanthappa and S. Oh, Phys. Rev. D62, 015012 (2000); R. Casadio, A. Gruppuso, and G. Venturi, Phys. Lett. B495, 378 (2000).

[13] L.E. Ibanez and C. Lopez, Nucl. Phys B233, 511 (1984); L.E. Ibanez, C. Lopez, and C. Munoz, Nucl. Phys. B256, 218 (1985).

[14] H.E. Haber, Perspectives on Higgs Physics II, Gordon L. Kane ed., World Scientific, Singapore, 1997; hep-ph/9707213.

[15] M. Elsing, http://delphiwww.cern.ch/ offline/physics_links/lepc.htm]

[16] CLEO collaboration, hep-ex/9908022.

[17] S. Bertolini, F. Borzumati, A. Masiero, and G. Ridolfi, Nucl. Phys. B353, 591 (1991).

[18] E. Gabrielli and U. Sarid, Phys. Rev. Lett. 79, 4752 (1997).

[19] J.L. Feng and T. Moroi, Phys. Rev. D61, 095004 (2000). 
[20] R. Bernabei et al., Phys. Lett. B480, 23 (2000).

[21] R. Abusaidi et al., Phys. Rev. Lett. 84, 5699 (2000).

[22] A. Bottino, F. Donato, N. Fornengo, and S. Scopel, Phys. Rev. D59, 095004 (1999); R. Arnowitt and P. Nath, Phys. Rev. D60, 044004 (1999).

[23] E. Accomando, R. Arnowitt, B. Dutta, and Y. Santoso, Nucl. Phys. B585, 124 (2000); R. Arnowitt, B. Dutta, and Y. Santoso, hep-ph/0005154. 